\title{
Incorporating lead education content into undergraduate nursing curriculum: Impact on knowledge and confidence
}

\author{
Tsu-Yin Wu*1, Lydia McBurrows², Jenni Hoffman², Sarah Lally ${ }^{3}$, Vedhika Raghunathan ${ }^{4}$ \\ ${ }^{1}$ School of Nursing \& Center for Health Disparities Innovations and Studies, Eastern Michigan University, USA

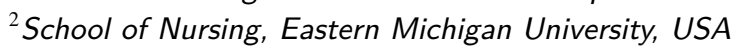 \\ ${ }^{3}$ Center for Health Disparities Innovations and Studies, Eastern Michigan University, Michigan Medicine, USA \\ ${ }^{4}$ College of Literature, Science, and the Arts, University of Michigan, USA
}

Received: July 24, 2021

DOI: $10.5430 /$ jnep.v12n1p56
Accepted: August 31, 2021

URL: https://doi.org/10.5430/jnep.v12n1p56

\begin{abstract}
Background and objective: Lead poisoning is a major public health crisis in Michigan. The purpose of this study was to explore the impact of an education intervention on knowledge and confidence levels among nursing students enrolled in the pre-licensure Bachelor of Science in Nursing and Registered Nurse to Bachelor of Science in Nursing (RN2BSN) program.

Methods: The study used a quantitative pre- and post-test design to assess the impact of lead health learning activities on knowledge and confidence among undergraduate nursing students in the Midwestern United States. The final study sample included 115 nursing students from two student cohorts. The study instrument used 26-item Nursing Students Lead Knowledge and Confidence Scale; independent sample $t$-tests, paired sample $t$-test and Cohen's $\mathrm{d}$ for the effect size were used in data analyses.

Results: The education improved total knowledge and confidence on both groups whereas RN2BSN students had larger effect sizes on the differences of pre- to post-test scores than pre-licensure students in general lead knowledge, lead exposure knowledge, total lead knowledge, and confidence.

Conclusions: The results contribute to limited literature examining a critical public health concern regarding lead health exposure and prevention education of nursing students. Incorporating such content area into nursing curriculums is essential in ensuring that such public health disparities are mitigated.
\end{abstract}

Key Words: Community health nursing, Lead poisoning, Lead testing

\section{INTRODUCTION}

Lead exposure accounted for 1.7 million deaths across the world. ${ }^{[1]}$ Lead is one of the most common environmental toxins for children less than six years old in the United States. ${ }^{[2]}$ Children and pregnant women are more susceptible to lead poisoning than other adults. ${ }^{[3]}$ Low socio-economic status, racial and ethnic groups, and immigrants are at greater risk for lead poisoning. ${ }^{[2]}$ Eliminating lead poisoning as a public health problem requires intervening on state-level risk factors and tailoring strategies in testing, reporting and surveillance as well as linkage to care.

In Michigan, there are several environmental risk factors for

\footnotetext{
*Correspondence: Tsu-Yin Wu; Email: twu@emich.edu; Address: School of Nursing \& Center for Health Disparities Innovations and Studies, Eastern Michigan University, USA.
} 
lead poisoning. In 2014 and 2018, the state of Michigan experienced two-large scale lead poisoning epidemics in Flint and Detroit related to lead contaminated water. The Flint water crisis in 2014 created increased overall awareness over lead exposure amongst children. This, combined with the discovery of elevated levels of lead in drinking water in Detroit public schools in 2018, has further highlighted this health crisis amongst children in Michigan. In Michigan, at least $50 \%$ of the homes in several communities like Detroit, Hamtramck, Highland Park, Grosse Pointe, and Wyandotte were built before 1950, putting these communities at increased risk for lead poisoning related to contamination from leadbased paint and fixtures. ${ }^{[4]}$ Approximately 58,565 children under the age of six years lived in Detroit in 2016 with $91.9 \%$ of the children in this age group living in homes built prior to 1980 , and $58.0 \%$ living in homes built prior to $1950 .^{[5]}$

In addition to high percentages of young children living in older homes, there are high percentages of children in Detroit and Michigan with elevated blood lead levels (EBLL) defined as $\geq 5 \mu \mathrm{g} / \mathrm{dL}$ of lead in blood. Michigan blood lead level data for 2016 showed that 5,724 (3.6\%) children in Michigan under age six years were diagnosed with EBLL; in Detroit, 2,073 (8.8\%) children under the age of six years had EBLL. ${ }^{[5]}$ It is believed the actual number of lead poisoned children is much higher than reported due to inadequate testing. ${ }^{[2,5]}$

Healthcare providers play an important role in preventing primary lead exposure, identifying children and pregnant women with a high risk for lead poisoning, and ensuring lead testing is completed. ${ }^{[6]}$ Medicaid providers are required by law to screen children for lead poisoning; however, only $20.9 \%$ of Michigan's total population under the age of six years were screened in 2016. ${ }^{[2]}$ Studies report insufficient training, knowledge deficits, and lack of confidence in screening for lead and lead treatment among healthcare providers..$^{[7,8]}$

Currently, there are limited studies that examine healthcare providers' lead knowledge and beliefs, and most studies were conducted over a decade ago. Therefore, it is important to evaluate the impact of learning activities designed for increasing awareness and knowledge of lead poisoning and prevention among nursing students as nurses play an essential role in educating families on how to prevent lead exposure. The purpose of this study was to strengthen community health content to address a timely public health crisis in undergraduate nursing curriculum. Research aims in this study were: 1) to examine the baseline lead knowledge and confidence between pre-licensure Bachelor of Science in Nursing (BSN) and Registered Nurse to BSN (RN2BSN)

Published by Sciedu Press students, and 2) to evaluate the impact of multi-modal education interventions and compare total knowledge score and three sub-areas of knowledge and confidence scores among pre-licensure BSN and RN2BSN students.

\section{METHODS}

\subsection{Study design, setting and participants}

The study used a quantitative pre- and post-test design to assess the impact of lead health learning activities on knowledge and confidence among undergraduate nursing students in Michigan. Eligible participants were in the pre-licensure BSN and RN2BSN programs, and taking a senior-level community health nursing course. The students received lead poisoning and prevention lecture content in addition to completing an e-learning module titled, "Pediatric Lead Exposure: Diagnosis, Management and Prevention" developed by the CDC and Pediatric Environmental Health Specialty Units. ${ }^{[9]}$ The lead health content aims to enhance students' knowledge and competence for the understanding, diagnosis, management, and prevention of pediatric lead poisoning as a result of environmental exposures.

The study consisted of 131 pre-licensure BSN and RN2BSN students; 16 students did not participate in both pre-test and post-test evaluation; therefore, they were excluded from the study. The final sample of the study consisted of 115 nursing students (55 pre-licensure BSN and 60 RN2BSN students) with a response rate $=88 \%$.

\subsection{Study procedures}

The study protocol was reviewed and approved by the University Institutional Review Board. Students' participation in the study was entirely voluntary and they could withdraw at any time without consequences. Participants provided consent, completed a pre-test one week before the lead health education and then completed a post-test one week after the education; participation occurred over the course of three weeks. Since one of the researchers was a community health nursing faculty, in order to prevent student participants from feeling coerced, the data was collected by two non-community health nursing faculty members of the research team during the fall 2019 semester in classrooms and collected online during the winter 2020 semester. Additionally, data were not analyzed until after course grades were submitted.

\subsection{Measurement}

The study instrument had two sections: 1) Demographics and 2) 26-item Nursing Students Lead Knowledge and Confidence Scale (NS-LEKS) that examined students' knowledge of lead poisoning and prevention, and how to identify children that are at risk of blood lead poisoning. The scale items 
were adapted from the Chicago Lead Knowledge Test ${ }^{[10]}$ and "What do you know?" a Chinese lead knowledge test developed by Huang and colleagues; ${ }^{[11]}$ the Pearson productmoment correlation for test scores was 0.96. The NS-LEKS has a true-false and Likert-scale format that measures general knowledge related to lead (six items), lead exposure (12 items), lead poisoning prevention (five items), and confidence in lead knowledge (three items). The scores for knowledge were calculated by the percentage of accuracy on all the items in the total knowledge scale and three knowledge dimensions, while the score for confidence was calculated using three items of the confidence subscale.

\subsection{Data analysis}

The data were analyzed using the IBM Statistical Package for the Social Sciences (SPSS) Statistics for Windows, Ver- sion 25. The statistical analyses were performed using 1) independent sample $t$-test for the comparisons between prelicensure and RN2BSN groups on baseline (i.e., pre-tests), 2) a paired sample $t$-test was for assessing differences between knowledge scores from pretest and posttest mean scores for both groups and 3) Cohen's $\mathrm{d}^{[12]}$ for the effect size.

\section{RESUltS}

\subsection{Demographics among study participants}

The majority $(87.8 \%)$ of the participants were females whereas the two groups of students had approximately the same distributions (see Table 1); $52.2 \%$ of participants were from the RN2BSN group and $47.8 \%$ were from the prelicensure group. Most of the participants reported being currently employed in healthcare $(76.7 \%$ ) (see Table 1 ).

Table 1. Demographic characteristics of participants

\begin{tabular}{|c|c|c|c|c|c|c|}
\hline \multirow{2}{*}{ Characteristics } & \multicolumn{2}{|c|}{ Overall Sample } & \multicolumn{2}{|c|}{ Pre-Licensure } & \multicolumn{2}{|c|}{ RN-to-BSN } \\
\hline & $\mathbf{N}$ & $\%$ & $\mathbf{N}$ & $\%$ & $\mathbf{N}$ & $\%$ \\
\hline Gender & 115 & & 55 & & 60 & \\
\hline Male & 13 & 11.3 & 7 & 12.7 & 6 & 10.0 \\
\hline Female & 101 & 87.8 & 48 & 87.3 & 53 & 88.3 \\
\hline Prefer not to answer & 1 & 0.9 & 0 & 0.0 & 1 & 1.7 \\
\hline Nursing program type & 115 & & 55 & & 60 & \\
\hline BSN Traditional & 1 & 0.9 & 1 & 1.8 & 0 & 0.0 \\
\hline BSN Second degree & 34 & 29.6 & 34 & 61.8 & 0 & 0.0 \\
\hline BSN Collaborative & 20 & 17.4 & 20 & 36.4 & 0 & 0.0 \\
\hline RN2BSN & 60 & 52.2 & 0 & 0.0 & 60 & 100.0 \\
\hline Current employment in healthcare & 116 & & 55 & & 60 & \\
\hline Yes & 89 & 76.7 & 33 & 60.0 & 56 & 93.3 \\
\hline No & 26 & 22.4 & 22 & 40.0 & 4 & 6.7 \\
\hline
\end{tabular}

3.2 Baseline comparisons on lead knowledge and confidence

Independent sample $t$-tests were conducted for pre-test scores in general lead knowledge, lead exposure knowledge, lead poisoning prevention knowledge, and total lead knowledge between pre-licensure and RN2BSN students. Although the RN2BSN group reported higher scores than the pre-licensure group on overall knowledge as well as general lead knowledge, lead prevention knowledge, and confidence dimensions, there were no statistical differences on all the scores tested among these two nursing student groups (see Table 2).

\subsection{Impact of learning activities on lead knowledge and confidence}

Paired sample $t$-tests and Cohen's d effect size were performed to examine the effects of lead learning activities on total lead knowledge, three lead knowledge dimensions, and confidence among two groups. Amongst pre-licensure stu- dents, pre- and post-test results in prevention knowledge $(\mathrm{t}(\mathrm{df})=-3.542(51), p=.001, \mathrm{~d}=0.49)$, total knowledge $(\mathrm{t}(\mathrm{df})$ $=-3.794(48), p=.000, \mathrm{~d}=0.54)$, and confidence $(\mathrm{t}(\mathrm{df})=$ $-3.987(51), p=.000 . \mathrm{d}=0.55)$ were statistically significant with small and medium effect sizes respectively.

Amongst RN2BSN students, pre- and post-test results were statistically different across general knowledge $(\mathrm{t}(\mathrm{df})=$ 2.209(52), $p=.032, \mathrm{~d}=0.30)$ with small effect size, exposure knowledge $(\mathrm{t}(\mathrm{df})=-3.529(53), p=.001, \mathrm{~d}=0.49)$ with medium effect size, prevention knowledge $(\mathrm{t}(\mathrm{df})=$ $3.268(53), p=.002, \mathrm{~d}=0.44)$ with medium effect size, total knowledge $(\mathrm{t}(\mathrm{df})=-4.696(52), p=.000, \mathrm{~d}=0.64)$ with medium effect size, and confidence $(\mathrm{t}(\mathrm{df})=-8.625(53), p=$ $.000, \mathrm{~d}=1.170$ ) with a large effect size. RN2BSN students had larger effect sizes on the differences of pre- to post-test scores than pre-licensure students in the following areas: general lead knowledge, lead exposure knowledge, total lead knowledge, and confidence. In lead prevention knowledge, 
pre-licensure students had a slightly larger Cohen's d ( $\mathrm{d}=$ $0.49)$ compared to RN2BSN students $(\mathrm{d}=0.44)$ (see Table 3).

Independent sample $t$-tests were conducted to examine the differences of pre- and post-test scores among two student groups. While RN2BSN students reported higher increases in total knowledge, lead exposure, and confidence dimensions, pre-licensure students reported higher increases in general lead and lead prevention knowledge; these differences did not reach statistical significance (see Table 4).

Table 2. Total knowledge and three sub-areas (general, lead exposure, and prevention knowledge) on the baseline between pre-licensure nursing and RN2BSN students

\begin{tabular}{|c|c|c|c|c|c|c|}
\hline Knowledge category & $\mathbf{N}$ & Mean (SD) & $\mathbf{t}(\mathbf{d f})$ & $p$-value & $\begin{array}{l}95 \% \text { CI of the } \\
\text { difference } \\
\text { lower bound }\end{array}$ & $\begin{array}{l}95 \% \text { CI of the } \\
\text { difference } \\
\text { upper bound }\end{array}$ \\
\hline $\begin{array}{l}\text { General knowledge } \\
\text { Pre-licensure } \\
\text { RN2BSN }\end{array}$ & $\begin{array}{l}55 \\
59\end{array}$ & $\begin{array}{l}92.73(11.900) \\
93.50(9.795)\end{array}$ & $\begin{array}{l}-0.381 \\
(112)\end{array}$ & .704 & -4.809 & 3.258 \\
\hline $\begin{array}{l}\text { Exposure knowledge } \\
\text { Pre-licensure } \\
\text { RN2BSN }\end{array}$ & $\begin{array}{l}54 \\
60\end{array}$ & $\begin{array}{l}81.02(10.418) \\
79.86(10.326)\end{array}$ & $\begin{array}{l}0.595 \\
(112)\end{array}$ & .553 & -2.696 & 5.011 \\
\hline $\begin{array}{l}\text { Prevention knowledge } \\
\text { Pre-licensure } \\
\text { RN2BSN }\end{array}$ & $\begin{array}{l}55 \\
60\end{array}$ & $\begin{array}{l}72.36(19.433) \\
74.67(17.219)\end{array}$ & $\begin{array}{l}-0.674 \\
(113)\end{array}$ & .502 & -9.075 & 4.469 \\
\hline $\begin{array}{l}\text { Total knowledge } \\
\text { Pre-licensure } \\
\text { RN2BSN }\end{array}$ & $\begin{array}{l}54 \\
59\end{array}$ & $\begin{array}{l}82.05(9.020) \\
82.39(8.131)\end{array}$ & $\begin{array}{l}-0.212 \\
(111)\end{array}$ & .832 & -3.540 & 2.854 \\
\hline $\begin{array}{l}\text { Confidence } \\
\text { Pre-licensure } \\
\text { RN2BSN }\end{array}$ & $\begin{array}{l}55 \\
60\end{array}$ & $\begin{array}{l}61.70(15.984) \\
62.67(13.734)\end{array}$ & $\begin{array}{l}-0.350 \\
(113)\end{array}$ & .727 & -6.463 & 4.523 \\
\hline
\end{tabular}

Note. CI: confidence interval; *Statistically significant $(p<.05)$

Table 3. Paired samplet-test results of pre- and post-test scores for pre-licensure and RN2BSN students

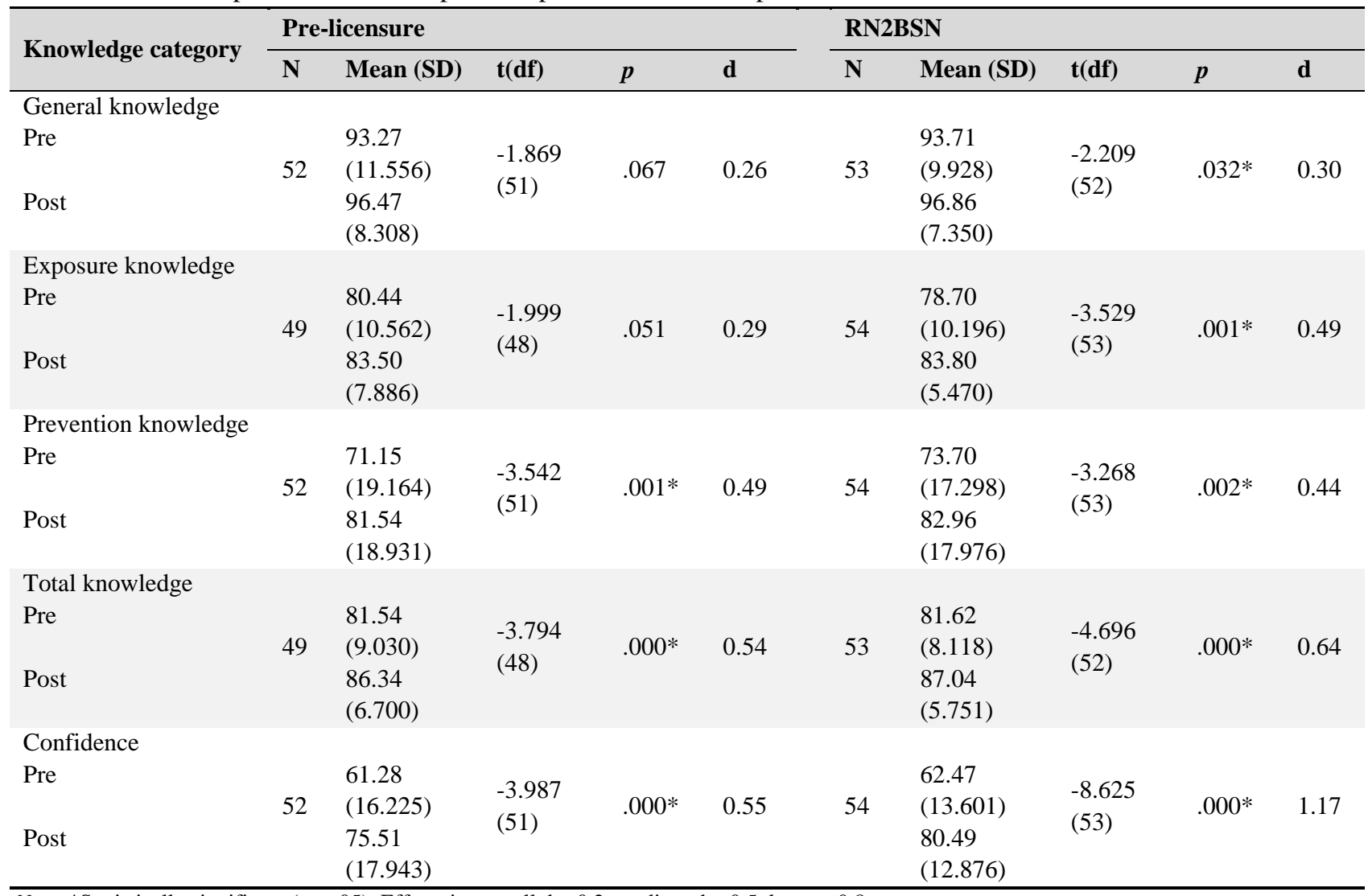

Note. $*$ Statistically significant $(p<.05)$; Effect size: small $\mathrm{d}=0.2$, medium $\mathrm{d}=0.5$, large $=0.8$. 
Table 4. Differences in pre- and post-test scores on total knowledge and three dimensions of knowledge and confidence between pre-licensure and RN2BSN students

\begin{tabular}{|c|c|c|c|c|c|c|}
\hline Knowledge category & $\mathbf{N}$ & Mean (SD) & $t(d f)$ & $p$-value & $\begin{array}{l}95 \% \text { CI of the } \\
\text { difference lower } \\
\text { bound }\end{array}$ & $\begin{array}{l}95 \% \text { CI of the } \\
\text { difference } \\
\text { upper bound } \\
\end{array}$ \\
\hline Total knowledge & & & \multirow{3}{*}{$\begin{array}{l}0.027 \\
(103)\end{array}$} & \multirow{3}{*}{.978} & \multirow{3}{*}{-4.353} & \multirow{3}{*}{4.474} \\
\hline Pre-licensure & 52 & $3.21(12.366)$ & & & & \\
\hline RN2BSN & 53 & $3.14(10.365)$ & & & & \\
\hline Exposure knowledge & & & \multirow{3}{*}{$\begin{array}{l}-0.975 \\
(101)\end{array}$} & \multirow{3}{*}{.332} & \multirow{3}{*}{-6.165} & \multirow{3}{*}{2.102} \\
\hline Pre-licensure & 49 & 3.06 (10.717) & & & & \\
\hline RN2BSN & 54 & 5.09 (10.418) & & & & \\
\hline Prevention knowledge & & & \multirow{3}{*}{$\begin{array}{l}0.276 \\
(104)\end{array}$} & \multirow{3}{*}{.783} & \multirow{3}{*}{-6.957} & \multirow{3}{*}{9.207} \\
\hline Pre-licensure & 52 & $10.38(21.140)$ & & & & \\
\hline RN2BSN & 54 & $9.26(20.818)$ & & & & \\
\hline Total knowledge & & & \multirow{3}{*}{$\begin{array}{l}-0.365 \\
(100)\end{array}$} & \multirow{3}{*}{.716} & \multirow{3}{*}{-4.009} & \multirow{3}{*}{2.763} \\
\hline Pre-licensure & 49 & 4.79 (8.841) & & & & \\
\hline RN2BSN & 53 & $5.41(8.394)$ & & & & \\
\hline Confidence & & & \multirow{3}{*}{$\begin{array}{l}-0.917 \\
(82.624)\end{array}$} & \multirow{3}{*}{.362} & \multirow{3}{*}{-12.021} & \multirow{3}{*}{4.433} \\
\hline Pre-licensure & 52 & $14.23(25.736)$ & & & & \\
\hline RN2BSN & 54 & $18.02(15.358)$ & & & & \\
\hline
\end{tabular}

Note. CI: confidence interval; *Statistically significant $(p<.05)$.

\section{Discussion}

Lead exposure is a critical health disparity faced by children in Michigan; nevertheless, the existing literature around this topic has been largely outdated. Health professionals play a critical role in decreasing the risk of lead exposure and poisoning amongst at-risk populations. The results of the current study showed that there were statistically significant increases in lead health knowledge and confidence after lead education in a community health nursing course and no differences were detected between pre-licensure and RN2BSN nursing students in pre-tests and also knowledge gained scores (i.e., difference between pre- to post-tests) across general lead, lead exposure, lead prevention, total lead knowledge, and confidence scores. Thus, despite RN2BSN students already having an active RN license, the levels of knowledge in lead exposure and prevention, and confidence were approximately equivalent to pre-licensure students.

While current clinical guidelines highlight the importance of lead testing and patient education in preventing and managing lead exposure, existing literature has shown that gaps in knowledge, practice, and training still exist amongst nurses and other healthcare providers, ${ }^{[7,8,13]}$ nursing students, and psychologists. ${ }^{[14,15]}$ Kilpatrick et al. (2002) found that surveyed pediatricians reported lower self-reported efficacy in environmental history-taking, discussing environmental exposures with parents, and locating diagnosis and treatment resources related to environmental exposures. Similarly, another study surveying pediatricians in Michigan found that only $15.4 \%$ of respondents reported having any training in environmental history taking and $78.1 \%$ reported being interested in additional training in this area. ${ }^{[8]}$ A more recent study ${ }^{[14]}$ assessing nursing students found that there were certain knowledge gaps in signs of illness, risk factors, and behavioral impacts of lead poisoning, preventative measures, and medications for treating lead poisoning. These topics were identified as important areas in the current study and have been incorporated into community health nursing didactic content. Future studies are warranted to examine the effectiveness of continuing education programs to strengthen the knowledge base on lead poisoning prevention and early intervention for healthcare providers and nursing students.

The current study utilized a self-learning module for lead education. A recent study conducted amongst first year undergraduate nursing students found that self-learning modules helped facilitate the learning and practice of their nursing clinical skills and increase their clinical competency. ${ }^{[16]}$ Incorporating such self-learning educational tools during the COVID-19 pandemic can be useful while in-person instructions are limited. Tools such as the Pediatric Lead Assessment Network Education Training (PLANET) and other selflearning modules such as the one developed in the current study can be incorporated into continuing education curriculum to effectively train practicing nurses.

Nurses are in a unique and salient position for patient advocacy and education; as a result, educating nurses to improve their knowledge and practices about lead exposure and prevention in children, particularly in vulnerable areas such as 
Detroit, is of utmost importance. Education should highlight existing health disparities and inequalities in vulnerable high-risk populations related to lead poisoning and other environmental health issues. With thorough lead health education and training, nurses will have increased confidence and skills to assess and screen for lead poisoning risk factors and to effectively link underserved populations with community resources for mitigating elevated lead levels.

\section{Limitations}

Although there was a lack of randomization, the similarities on the scores in knowledge and confidence among two study groups (i.e., pre-licensure vs. RN2BSN students) at the baseline enhance the study's internal validity. Several study limitations were noted. The study was based on only a small convenience sample which limits the generalizability. Since the pre- and post-test consisted of the same questions, participants may have remembered some of the correct answers from the pre-test and therefore, this may have resulted in higher scores on the post-test. The study also did not measure knowledge retention for long-term effects; as a result, students may not be able to recall lead health content after periods of time. Nevertheless, the significant increase in scores between the pre-test and post-test in both groups suggests that lead educational components increase the stu- dents' knowledge acquisition and improves their confidence to provide lead health-related education for their patients.

\section{Conclusion}

Lead poisoning is a critical public health issue that greatly affects underserved populations. Given the prevalence and severity of lead exposure in certain geographic areas with underserved populations in Michigan, educating nurses and other healthcare providers on best practices to address lead exposure is critical. Such intervention effort early in the education curriculum has the potential to lessen health disparities in lead exposure amongst children. The results of the study suggest that undertaking multi-modal lead education could be a useful addition to pedagogy for teaching nursing students about lead poisoning. In summary, the results contribute to an emerging body of literature examining the lead health exposure and prevention knowledge of nursing students.

\section{ACKNOWLEDgements}

The authors are grateful to Dr. Marty Raymond for his assistance in data management and Angela Medina for her input in the process of developing lead education intervention.

\section{Conflicts of InTEREST Disclosure}

The authors declare that there is no conflict of interest.

\section{REFERENCES}

[1] World Health Organization. Lead poisoning and health. 2019. Available from: https://www.who.int/news-room/fact-sheets/ detail/lead-poisoning-and-health

[2] Centers for Disease Control and Prevention. CDC National childhood blood lead surveillance data. 2019. Available from: https: //www.cdc.gov/nceh/lead/data/national.htm

[3] United States Environmental Protection Agency. Learn about lead. 2019. Available from: https://www. epa.gov/lead/learn-abo ut-lead\#exposed

[4] Drawing Detroit. Monthly archives: November 2017 Wayne County Home to Region's Oldest Homes. 2017. Available from: http: //www.drawingdetroit.com/2017/11/

[5] Michigan Childhood Lead Poisoning Prevention Program, Michigan Department of Health and Human Services Division of Environmental Health. 2016 Data Report on Childhood Lead Testing and Elevated Levels: Michigan. 2017. Available from: https://www.michigan .gov/lead/0, 5417, 7-310-84214--- , 00.html

[6] Michigan Department of Health and Human Services. (n.d.). Lead poisoning prevention for healthcare providers. Available from: https://www.michigan.gov/lead/0, 5417, 7-310-8 4217--, 00.html

[7] Choate D, Polivka B. Ohio APNs: Lead poisoning knowledge and practices. Journal of the American Academy of Nurse Practitioners. 2000; 12(12): 503-510. PMid:11930596 https://doi.org/10.1 $111 / j .1745-7599 \cdot 2000 . t b 00165 . x$
[8] Trasande L, Newman N, Long L, et al. Translating knowledge about environmental health to practitioners: Are we doing enough? Mount Sinai Journal of Medicine. 2010; 77: 114-123. PMid:20101722 https://doi.org/10.1002/ms j. 20158

[9] Pediatric Environmental Health Specialty Units. Pediatric Lead Exposure: Diagnosis, Management and Prevention. 2017. Available from: https://www.pehsuclassroom.net/learn/course/ex ternal/view/elearning/77/pediatric-lead-exposure-d iagnosis-management-and-prevention

[10] Rabito FA, Sarpy SA, Shorter C, et al. Lead prevention knowledge among low-income urban residents: The Chicago lead knowledge test revisited. Journal of Children's Health. 2004; 2(1): 11-20. https://doi.org/10.3109/15417060490453236

[11] Huang R, Ning H, Baum CR, et al. "What do you know?"-knowledge among village doctors of lead poisoning in children in rural China. BMC Public Health. 2017; 17(1): 895-899. PMid:29169343 https : //doi.org/10.1186/s12889-017-4895-2

[12] Cohen J. Statistical Power Analysis for the Behavioral Sciences. Mahwah, NJ: Lawrence Erlbaum Associates. 1998.

[13] Kilpatrick N, Frumkin H, Trowbridge J, et al. The environmental history in pediatric practice: a study of pediatricians' attitudes, beliefs, and practices. Environmental Health Perspectives. 2002; 110(8): 823 827. PMid:12153766 https://doi.org/10.1289/ehp.021108 23

[14] Morrison S, Ressler E, Sheets K, et al. Nursing Students' Perception of Childhood Lead Poisoning Risk Factors. International Journal of 
Studies in Nursing. 2017; 2(2): 37-43. https ://doi.org/10. 208 49/ijsn.v2i2.216

[15] Finlay JT. Perceptions of Child Lead Poisoning Among Educational Psychologists (Publication No. 2827941) [Masters dissertation, University of Johannesburg]. ProQuest Dissertations Publishing. 2019.
[16] Tohidi S, KarimiMoonaghi H, Shayan A, et al. The Effect of Selflearning Module on Nursing Students' Clinical Competency: A Pilot Study. Iranian Journal of Nursing and Midwifery Research. 2019; 24(2): 91-95. PMid:30820218 https://doi.org/10.4103/ijnm r. IJNMR_46_17 